\title{
平原河湖系统中典型全氟化合物的胶体吸附特征及生态风险评估”
}

\author{
刘建超 ${ }^{1}$, 郑超亚 ${ }^{1}$, 任静华 ${ }^{2 * *}$, 陆光华 $^{1}$, 李一平 $^{1}$ \\ (1:河海大学环境学院,浅水湖泊综合治理与资源开发教育部重点实验室,南京 210098) \\ ( 2 : 江苏省地质调查研究院,自然资源部 (国土) 耕地生态监测与修复工程技术创新中心,南京 210018)
}

\begin{abstract}
摘 要: 胶体作为水环境中广泛存在的一类纳米颗粒物, 因其粒径小、比表面积大、吸附位点多等特点, 能够吸附有机污 染物,并改变和控制其环境行为. 全氟化合物 (PFCs) 疏水疏油、环境持久性强,在水环境中势必与胶体介质结合,形成复 合污染效应. 本文以不同受纳河湖系统作为研究区域, 选取 4 种典型的 PFCs 为目标物, 主要包括: 全氟辛酸 (PFOA)、全 氟壬酸( PFNA)、全氟辛烷磺酸(PFOS)、全氟正癸酸(PFDA), 研究其在传统溶解相中的空间分布特征及胶体吸附潜能, 并评估其生态风险水平, 主要结果如下: (1) 4 种 PFCs 在研究区域传统溶解相和胶体相中均被检出, 检出率为 $78.8 \%$ $100 \%$, 总浓度范围分别在 12.5 92.1 和 5.4 49.9 ng/L 之间. 在传统溶解相和胶体相中, PFOA 均为最主要的 PFCs, 贡献 率在 65\% 以上, 其次是 PFOS、PFNA 和 PFDA. (2) 从介质分布来看, 胶体是 PFCs 的重要吸附载体, 平均吸附贡献率在 $42.6 \% \sim 66.1 \%$ 之间, 其中胶体对 PFDA 的吸附潜能最大, 平均吸附贡献率为 $66.1 \%$, 其次是 PFNA ( 53.5\%)、PFOA $(47.0 \%)$ 和 PFOS $(42.6 \%)$, 胶体对 PFCs 的吸附贡献率与其正辛醇一水分配系数 ( $\left.\lg K_{O W}\right)$ 呈显著正相关. (3) 从空间分布 来看, PFCs 在居民聚集区污染水平最高, 平均浓度为 $71.2 \mathrm{ng} / \mathrm{L}$, 其次是溱湖湿地公园, 平均浓度为 $43.3 \mathrm{ng} / \mathrm{L}$. 工业区、农 业区及城市区, PFCs 污染水平相当, 平均浓度在 30 40 ng/L 之间, 水产养殖区污染水平最低. (4) 通过风险熵值法 $(R Q)$ 和水环境质量基准对检出 PFCs 进行风险评价, 发现 58\% 水域中的水生生物处于 PFOS 引起的低风险状态, 主要集中于工 业区以及居民聚集区. 且所有采样点中 PFOS 的污染水平均高于欧盟委员会设定的 PFOS 在淡水环境中的年均环境质量 标准 $(0.65 \mathrm{ng} / \mathrm{L})$, 低于最大允许浓度 $36 \mu \mathrm{g} / \mathrm{L}$. PFOS 在水环境长期赋存引起的慢性毒理风险不容忽视.
\end{abstract}

关键词: 全氟化合物; 河湖系统;赋存;胶体;生态风险;溱湖湿地公园

\section{Adsorption characteristics of perfluorinated compounds from colloids in the river-lake sys- tem of the plain and their ecological risk*}

Liu Jianchao $^{1}$, Zheng Chaoya ${ }^{1}$, Ren Jinghua ${ }^{2 * *}$, Lu Guanghua ${ }^{1} \&$ Li Yiping $^{1}$

(1: Key Laboratory for Integrated Regulation and Resources Development on Shallow Lakes, Ministry of Education, College of Environment, Hohai University, Nanjing 210098, P.R.China)

(2: Technology Innovation Center of Ecological Monitoring \& Restoration Project on Land (Arable), MNR Geological Survey of Jiangsu Province, Nanjing 210018, P.R. China)

Abstract: In the aquatic environment, colloids are ubiquitous and have small particle size, large specific surface areas and multiple adsorption sites. Colloids can adsorb the organic contaminants, and change and control their environmental behavior. Perfluorinated compounds (PFCs) may be associated with the colloids in surface water to form effects of combined pollution due to non-hydrophilic and non-lipophilic properties and persistence of PFCs. In this paper, river-lake system of a national ecological demonstration area was selected as the study area, spatial distribution characteristics and colloidal adsorption potential for 4 typical PFCs including pentadecafluorooctanoic acid (PFOA), perfluorononanoic acid (PFNA), perfluorooctane sulfonate (PFOS), perfluorooctanoic acid (PFDA) were investigated in the traditionally dissolved phase to assess their ecological risk level. The main results

* 2020-09-09 收稿;2021-03-25 收修改稿.

中央高校基本科研业务费项目 (B200202106)、“耕地污染调查成果在基本农田保护修复中的应用试点研究 (苏财 建[2017]123 号)、国家自然科学基金项目(52039003)和中国博士后科学基金项目(2018M630507，2019T120389) 联合资助.

** 通信作者; E-mail:renjinghuahua@163.com. 
were as follows: (1) All four PFCs were detected in the traditionally dissolved phase and colloidal phase, with the detection rate of $78.8 \%-100 \%$ and the total concentrations of $12.5-92.1 \mathrm{ng} / \mathrm{L}$ and $5.4-49.9 \mathrm{ng} / \mathrm{L}$, respectively. In the traditional dissolved phase and colloidal phase, PFOA are the main PFCs. The contribution rates of PFOA to PFCs were more than 65\%, followed by PFOS, PFNA, PFDA. (2) From the medium distribution, colloids are an important "sink" of PFCs, average adsorption contribution rates of colloids to PFCs are between $42.6 \%$ and $66.1 \%$. PFDA showed the greatest adsorption potential, with an average absorption contribution rate of $66.1 \%$, followed by PFNA $(53.5 \%)$, PFOA $(47.0 \%)$ and PFOS $(42.6 \%)$. The adsorption contribution rate of colloids to PFCs was significantly positive correlation with the $\lg K_{O W}$ of PFCs. (3) In terms of spatial distribution, residential areas have the highest pollution levels, with an average concentration of $71.2 \mathrm{ng} / \mathrm{L}$, followed by Qinhu Wetland Park (43.3 ng/L). In industrial, agricultural and urban areas, PFCs have comparable pollution levels, with average concentrations between $30 \mathrm{ng} / \mathrm{L}$ and $40 \mathrm{ng} / \mathrm{L}$. Aquaculture area showed the lowest pollution levels. (4) Using risk quotient $(R Q)$ and water quality benchmark to assess the water environment risk, we found that the aquatic organisms in $58 \%$ of sampling sites were in low-risk state caused by PFOS, mainly located in industrial areas and residential areas. And PFOS pollution levels in all the sampling sites were higher than the annual average environmental quality standard $(0.65 \mathrm{ng} / \mathrm{L})$ setting by the European Commission for PFOS in freshwater environments, which is lower than the maximum acceptable concentration environmental quality standard of $36 \mu \mathrm{g} / \mathrm{L}$. Chronic ecological risks caused by long-term occurrence of PFOS in aquatic environment should not be ignored.

Keywords: Perfluorinated compounds; river-lake system; occurrence; colloids; ecological risk; Qinhu Wetland Park

全氟化合物 (Perfluorinated compounds, PFCs) 是一类人工合成的新型持久性有机污染物, 具有一定的生 物累积性、环境持久性和长距离迁徙性,其在化工、电子、纺织、制药、航空、电镀和合成洗涤剂等方面被大量 使用 ${ }^{[1-3]}$. PFCs 能够通过多种途径进人环境, 在地表水 ${ }^{[4-5]}$ 、沉积物 ${ }^{[6]}$ 、土壤 ${ }^{[7]}$ 、野生动物 ${ }^{[8]}$ 甚至人体 ${ }^{[9]}$ 内广 泛赋存, 产生生殖毒性、神经毒性及致畸性, 威胁生态安全及人类健康 ${ }^{[10-12]}$. 作为两种主要的 PFCs, 全氟辛 烷磺酸 ( perfluorooctane sulfonate, PFOS) 和全氟辛酸 ( perfluorooctanoic acid, PFOA)生产量和使用量最大, 环 境检出最为频繁, 先后被列人斯德哥尔摩公约和美国环保署的限制生产排放协议 ${ }^{[13]}$. 为了满足生产需要, 包括中国在内的多个发展中国家仍在大量使用, 导致 PFCs 成为危害我国生态环境的重要污染物, 尤其是在 长江三角洲地区、珠江三角洲地区、中北部工业区等. 我国长江流域氟化工业发达, 该区域河流、湖泊以及水 库中 PFCs 均有高频次的检出, 检出浓度一般低于 $\mu \mathrm{g} / \mathrm{L}$ 水平. 例如, 长江重庆段 16 种 PFCs 的总浓度在 $1.54 \sim 61.93 \mathrm{ng} / \mathrm{L}(\text { 均值 } 23.94 \mathrm{ng} / \mathrm{L})^{[14]}$, 长江汉江段 11 种 $\mathrm{PFCs}$ 的总浓度为 $0.16 \sim 23.04 \mathrm{ng} / \mathrm{L}^{[15]}$, 长江中游 的武汉段、洞庭湖、鄱阳湖、洪湖等区域, 平均浓度达到了 $22.53 \mathrm{ng} / \mathrm{L}$ 以上 ${ }^{[16]}$, 到长江下游黄浦江水体中, 14 种 PFCs 浓度最高达到 $596.2 \mathrm{ng} / \mathrm{L}$ (平均浓度 $226.3 \mathrm{ng} / \mathrm{L}$ ) ${ }^{[17]}$. 整个长江流域 PFOS 都是最主要的 PFCs, 下游 水体污染程度明显高于上游. 流域对比来看, 长江流域中 PFCs 污染水平高于太湖流域 ${ }^{[18]}$ 、珠江流域 ${ }^{[19]}$ 和乌 梁素海流域 ${ }^{[20]}$, 与韩国 ${ }^{[21]}$ 、西班牙 ${ }^{[22]}$ 部分河湖水域中 PFCs 浓度相当. 现有的研究大多集中在大江大河水 体,平原地区不同受纳水体中 PFCs 的污染特征尚不明确.

目前 PFCs 在水环境介质中的调查主要集中在传统水相和沉积相, 对于胶体介质的吸附影响研究还相 当鿒乏. 胶体介质具有体积小、比表面积大、吸附位点多等特点, 能够有效吸附水中的有机化合物, 并对其迁 移、转化、生物有效性等环境行为产生重要影响 ${ }^{[23-24]}$. 长江滨岸水体中胶体对新兴有机污染物的吸附贡献率 可达 $42.3 \%$, 吸附作用与胶体的粒径、电位、类腐殖质等因素直接相关 ${ }^{[24]}$. 泰州市姜堰区地跨长江三角洲和 里下河平原, 区域内河流湖泊众多, 水产丰富, 水资源利用类型多变, 经济社会与环境保护协调发展, 是我国 建设的首批国家级生态示范区. 基于以上背景, 本研究以姜堰区为完整研究区域, 在农业区、工业区、养殖 区、湿地区、城市区、农村居民聚集区等不同受纳类型河湖水体中开展 4 种典型的 PFCs 的定性定量分析工 作, 探讨其污染水平、成分组成、空间分布和胶体吸附潜能, 并评价其生态风险, 以期为生态示范区的水环境 保护和有机污染物综合治理提供理论基础和参考依据.

\section{1 材料和方法}

\section{1 标准品和试剂}

4 种 PFCs 标准品全氟辛酸( PFOA) 、全氟壬酸 (PFNA)、全氟正癸酸 (PFDA) 和全氟辛烷磺酸 $($ PFOS ) 购 
买于 Sigma-Aldrich 试剂公司; 正己烷、二氯甲烷、乙腈、甲醇、丙酮均为色谱纯试剂, 购于美国 Merck 公司; Waters Oasis WAX 固相萃取小柱 (200 mg, $6 \mathrm{~mL}$ ), 购于美国 Waters 公司; 实验用水为 Milli-Q 纯净水.

\section{2 采样点布设}

在泰州市姜堰区共设置了 33 个采样点位, 采样时间为 2018 年 8 月, 采样时节为丰水期, 天气情况良好, 水温在 $25.0 \sim 28.6^{\circ} \mathrm{C}$ 之间, 每个点位采集水样 $2 \mathrm{~L}$, 平行样品 2 个. 采样点主要分布在 7 类区域, 分别为水产 养殖区 $(\mathrm{S} 1$ 和 S2) 、溱湖湿地公园 $(\mathrm{S} 3 \sim \mathrm{S} 5)$ 、农业区 $(\mathrm{S} 6 \sim \mathrm{S} 10)$ 、农业/居民混合区 $(\mathrm{S} 11 \sim \mathrm{S} 19)$ 、居民聚集区 $(\mathrm{S} 20 \sim \mathrm{S} 24)$ 、工业区 $(\mathrm{S} 25 \sim \mathrm{S} 29)$ 和城市区 $(\mathrm{S} 30 \sim \mathrm{S} 33)$, 具体见图 1.

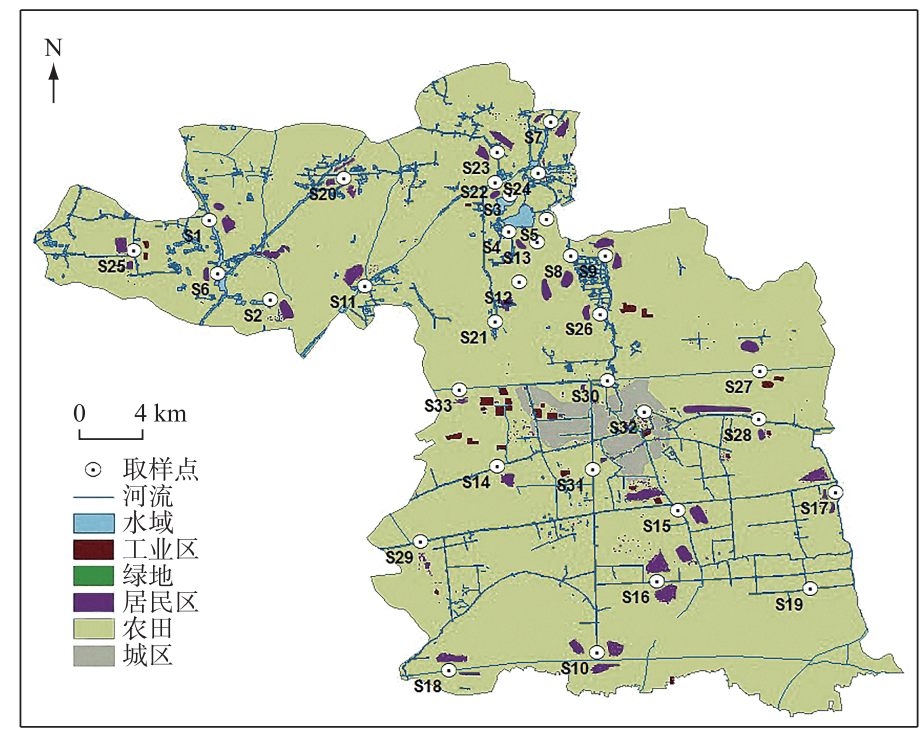

图 1 江苏省泰州市姜堰区采样点布设示意

Fig. 1 Distribution of sampling sites in Jiangyan District, Taizhou City, Jiangsu Province

\section{3 样品采集与前处理}

在水面以下 $0.3 \sim 0.5 \mathrm{~m}$ 范围内用准备好的聚丙烯采样瓶采集水样 $2 \mathrm{~L}$, 现场测定水质参数,采集后的水 样过 $1.0 \mu \mathrm{m}$ 的醋酸纤维滤膜. 将过滤后的水样利用切向超滤装置和孔径为 $1000 \mathrm{Da}$ 聚醚砜过滤膜进行分 离, 以获得胶体相 ( $1000 \mathrm{Da} \sim 1 \mu \mathrm{m}$ ) 和超滤水相 ( $<1000 \mathrm{Da})$. 胶体相和超滤水相加甲酸调至 $\mathrm{pH}=2.5$, 用固相

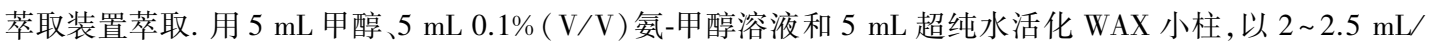
$\min$ 的速率过样, 经 $10 \mathrm{~mL}$ 超纯水进行淋洗. 最后用 $5 \mathrm{~mL}$ 甲醇和 $5 \mathrm{~mL} 0.1 \%(\mathrm{~V} / \mathrm{V})$ 氨-甲醇溶液进行洗脱, 洗 脱液被收集到聚丙烯管中, 氮吹至近干, 用甲醇定容至 $1 \mathrm{~mL}$, 然后过 $0.22 \mu \mathrm{m}$ 滤膜, 置于聚丙烯材质进样瓶 中, 低温冷藏待测.

表 1 流动相梯度变化

Tab.1 Gradient change of mobile phase

\begin{tabular}{ccc}
\hline 时间 $/ \mathrm{min}$ & 流动相 $\mathrm{A} / \%$ & 流动相 $\mathrm{B} / \%$ \\
\hline 0 & 90 & 10 \\
0.20 & 90 & 10 \\
4.00 & 5 & 95 \\
5.00 & 5 & 95 \\
5.01 & 90 & 10 \\
6.00 & 90 & 10 \\
\hline
\end{tabular}

\section{4 仪器分析与质量控制}

超高效液相色谱-三重四级杆串联质谱仪 (配有电喷 雾离子源)，Waters ACQUITY UPLC Xevo TQ, 美国 Waters 公司;ACQUITY BEH C18 色谱柱 $(2.1 \mathrm{~mm} \times 100 \mathrm{~mm}, 1.7$ $\mu \mathrm{m}$, 美国 Waters 公司). 流动相 $\mathrm{A}$ 为 $5 \mathrm{mmol} / \mathrm{L}$ 的醋酸铵 溶液, 流动相 $\mathrm{B}$ 为 $100 \%$ 乙腈; 流动相梯度变化如表 1 所示.

采用正负离子模式的电喷雾 $(\mathrm{ESI})$ 离子源以及多反 应监测模式 (MRM) 定量分析目标污染物. 质谱仪条件: 毛细管电压 $3.0 \mathrm{kV}$, 碰撞气体流速 $0.16 \mathrm{~L} / \mathrm{min}$, 离子源温 
度 $150^{\circ} \mathrm{C}$; 雉孔反吹气流量 $50 \mathrm{~L} / \mathrm{h}$; 脱溶剂气温度 $400^{\circ} \mathrm{C}$; 脱溶剂气流量 $900 \mathrm{~L} / \mathrm{h}$ 目标微污染物的电离模式、 母离子、子离子、碰撞能等质谱条件见表 2 .

表 2 目标化合物质谱参数

Tab.2 Mass spectrum parameters of the target compounds

\begin{tabular}{lcccccc}
\hline 化合物 & 缩写 & ESI & 母离子 $/(\mathrm{m} / \mathrm{z})$ & 子离子 $/(\mathrm{m} / \mathrm{z})$ & 雉孔电压 $/ \mathrm{V}$ & 碰撞能 $/ \mathrm{eV}$ \\
\hline 全氟辛酸 & PFOA & ESI+ & 413 & $219 / 369$ & 16 & $17 / 10$ \\
全氟壬酸 & PFNA & ESI+ & 463 & $169 / 219$ & 16 & $19 / 17$ \\
全氟辛烷磺酸 & PFOS & ESI+ & 499 & $80 / 99$ & 60 & $39 / 38$ \\
全氟癸酸 & PFDA & ESI+ & 513 & $219 / 469$ & 16 & $17 / 10$ \\
\hline
\end{tabular}

样品采集、样品前处理及样品分析整个过程遵循严格的质量控制程序. 采样过程设置野外现场空白. 每 批样品添加一个方法空白、溶剂空白和空白加标样进行实时校正. 空白组的基质为去离子水, 在现场空白、 方法空白和溶剂空白中 4 种 PFCs 目标物均未检出或低于定量限. 标准曲线的浓度范围为 $0.1 \sim 200 \mu \mathrm{g} / \mathrm{L}$, 线 性相关系数 $R^{2}>0.99$, 以 3 倍信噪比 ( $\mathrm{S} / \mathrm{N}$ ) 确定方法检出限 ( LOD ), 4 种 PFCs 的 LODs 在 $8.3 \sim 30.0 \mathrm{ng} / \mathrm{L}$ 之 间,在 $100 \mathrm{ng} / \mathrm{L}$ 浓度水平下的加标回收率范围为 $68.9 \% \sim 108.5 \%$,满足分析要求.

\section{5 数据分析方法}

为评估水体中 PFCs 对水生生物的影响, 采用风险熵值法 $(R Q)$ 对 PFOA、PFNA、PFOS 和 PFDA 进行生 态风险评价, $R Q$ 的计算方法为:

$$
R Q=M E C / P N E C
$$

式中, $M E C$ 和 $P N E C$ 分别为环境介质中实测浓度和预测无效应浓度. 根据所选毒性数据和模式生物营养级 的不同, $P N E C$ 需用不同的评估因子 $(A F)$ 计算得到. 采用急性毒性数据 $\mathrm{EC}_{50}$ 或 $\mathrm{LC}_{50}$ 前提下, $A F$ 取值为 1000 ; 当选用长期或慢性无可观察效应浓度 (NOEC) 时, 根据生物的营养级水平分别选取 $A F$ 值为 $100 、 50$ 和 10 . 由于慢性毒性数据缺失和有限的急性毒理数据, 综合考虑毒性试验的标准程度和毒性终点的敏感性, PNEC 采用评价因子法和基于物种敏感性分布的统计外推法获得, PFOA、PFNA、PFOS、PFDA 对于藻类、溞类、鱼类 的部分毒理数据见表 $3^{[25]}$. 通常按 $R Q$ 的大小将污染物的生态风险水平分为 4 个级别, $>1$ 为高风险, $0.1 \sim 1$ 之间为中等风险, $0.01 \sim 0.1$ 之间为低风险, $<0.01$ 为无风险.

表 34 种 PFCs 对水生生物的毒理数据

Tab.3 Toxicological data of four PFCs for aquatic organisms

\begin{tabular}{ccccc}
\hline 化合物 & $E C_{50 \text { 藻类 }} /(\mu \mathrm{g} / \mathrm{L})$ & $E C_{50 \text { 渭类 }} /(\mu \mathrm{g} / \mathrm{L})$ & $E C_{50 \text { 鱼类 }} /(\mu \mathrm{g} / \mathrm{L})$ & $A F$ \\
\hline PFOA & 748098 & 207000 & 260820 & 1000 \\
PFNA & 481632 & 92800 & 120640 & 1000 \\
PFOS & 23640 & 37360 & 3640 & 1000 \\
PFDA & 437414 & 77100 & 35980 & 1000 \\
\hline
\end{tabular}

\section{2 结果与讨论}

\subsection{PFCs 在传统水相中的分布特征}

传统溶解相中 4 种 PFCs 的污染水平及空间分布特征见图 2 和表 4. 从结果可知, 4 种 PFCs 中, PFOA、 PFNA 和 PFOS 的检测率最高, 为 100\%, 而 PFDA 则为 78.8\% . 4 种 PFCs 的总浓度在 12.5 92.1 ng/L 之间, 平均浓度为 $41.6 \mathrm{ng} / \mathrm{L}$. PFOA 作为最主要的污染物, 其浓度范围为 $11.5 \sim 69.3 \mathrm{ng} / \mathrm{L}$, 平均浓度为 $28.7 \mathrm{ng} / \mathrm{L}$. PFOS 和 PFNA 浓度相当, 最高浓度分别达到 21.0 和 $20.1 \mathrm{ng} / \mathrm{L}$, 平均浓度分别为 7.7 和 $3.6 \mathrm{ng} / \mathrm{L}$. PFDA 浓度 最低, 平均浓度为 $1.6 \mathrm{ng} / \mathrm{L}$, 最高达到 $13.0 \mathrm{ng} / \mathrm{L}$. 从单体贡献率上来看, PFOA 浓度占所有 PFCs 检出总浓度 的 70\% 以上, 其次是 PFOS(平均贡献率为 $18.5 \%$ )、 $\mathrm{PFNA}(7.8 \%)$ 和 $\operatorname{PFDA}(2.8 \%)$. 相似的结果在我国千岛 湖表层水和胶州湾表层海水中被发现, 在这两个区域中 PFOA 是众多 PFCs 中检测频率和检出浓度最高的 
污染物, 接近 $100 \%$, 在胶州湾海水中 $\mathrm{PFCs}$ 的总浓度在 $1.71 \sim 48.27 \mathrm{ng} / \mathrm{L}$ 之间, PFOA 平均浓度为 $16.41 \mathrm{ng} / \mathrm{L}$, 单体贡献率在 $29.2 \% \sim 95.6 \%$ 之间 ${ }^{[26-27]}$. 这与 PFCs 的市场使用量和使用途径直接相关, PFOA 和 PFOS 被广泛应用于石化行业、五金电镀、纺织品生产、橡胶乳化等工业制造和食品包装、纸类表面处理等生 活用品生产, 而 PFNA 和 PFDA 主要源于全氟羧酸生产过程的排放, 使用量和使用途径相对偏低.

表 4 姜堰区地表水中 PFCs 的浓度范围和平均值( 中值)

Tab.4 Concentration ranges and mean (median) values of PFCs in surface water from Jiangyan Districty

\begin{tabular}{|c|c|c|c|c|}
\hline \multirow{2}{*}{ 化合物 } & \multicolumn{2}{|c|}{ 传统溶解相 } & \multicolumn{2}{|c|}{ 胶体相 } \\
\hline & 浓度/(ng/L) & 检出频率/\% & 浓度/(ng/L) & 检出频率/\% \\
\hline PFOA & $\begin{array}{r}11.5 \sim 69.3 \\
28.7(25.7)\end{array}$ & 100 & $\begin{array}{c}2.4 \sim 28.0 \\
11.7(10.9)\end{array}$ & 100 \\
\hline PFNA & $\begin{array}{l}0.3 \sim 20.1 \\
3.6(2.6)\end{array}$ & 100 & $\begin{array}{c}0 \sim 20.1 \\
2.2(2.1)\end{array}$ & 97.0 \\
\hline PFOS & $\begin{array}{l}0.6 \sim 21.0 \\
7.7(7.3)\end{array}$ & 100 & $\begin{array}{l}0.2 \sim 10.2 \\
3.5(3.0)\end{array}$ & 100 \\
\hline PFDA & $\begin{array}{c}0 \sim 13.0 \\
1.6(0.4)\end{array}$ & 78.8 & $\begin{array}{c}0 \sim 8.9 \\
1.0(0.2)\end{array}$ & 78.8 \\
\hline
\end{tabular}

PFOA $\square$ PFNA $\square$ PFOS $\square$ PFDA

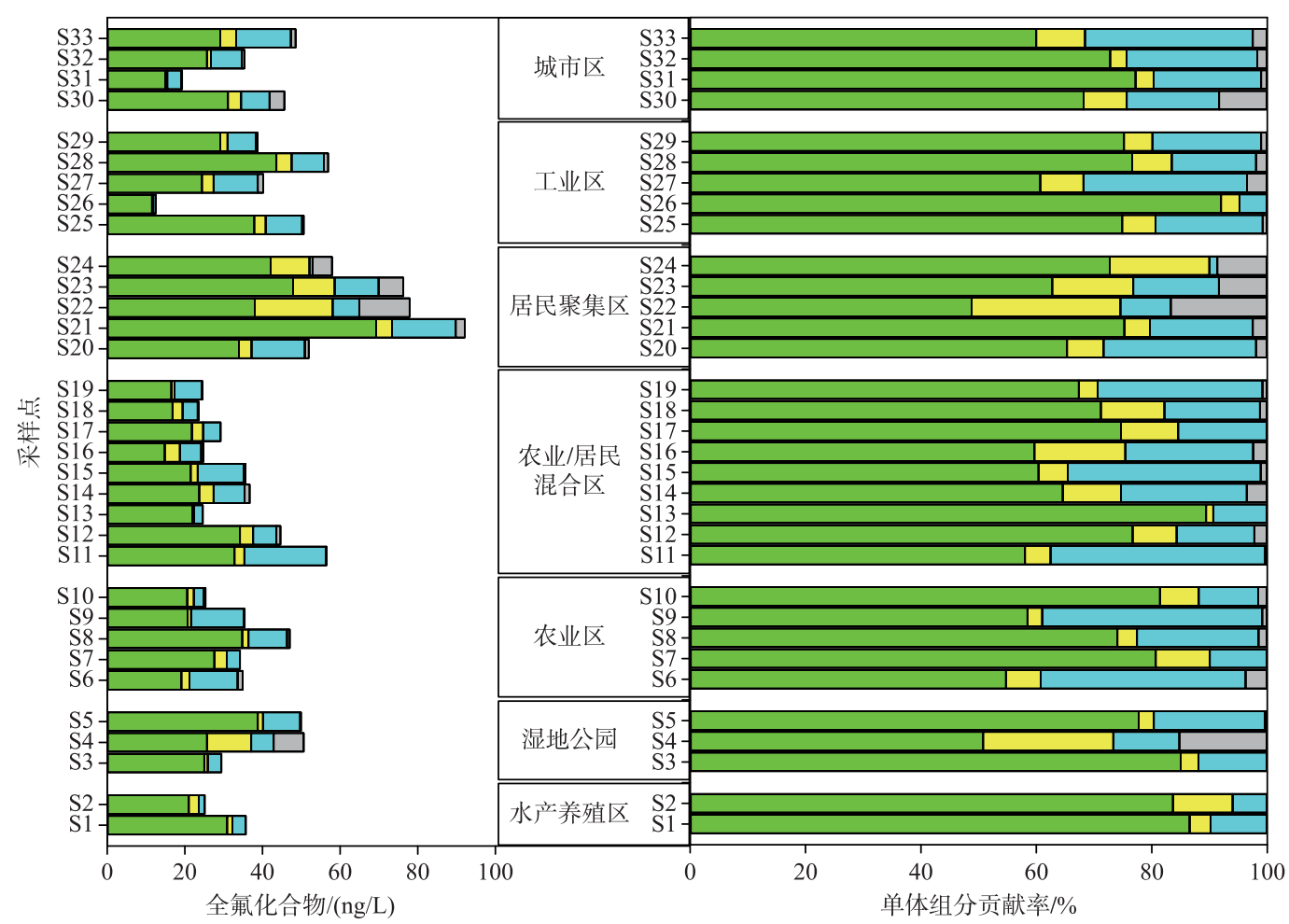

图 2 PFCs 在姜堰区地表水传统溶解相中的浓度及组分贡献率

Fig. 2 Concentrations and compositions of PFCs in traditionally dissolved phase of surface water from Jiangyan District

从空间分布来看, 居民聚集区 (S20 S24) 污染水平最高, 5 个采样点的 PFCs 总浓度范围为 51.9 92.1 
$\mathrm{ng} / \mathrm{L}$, 平均浓度为 $71.2 \mathrm{ng} / \mathrm{L}$. 其中泰东河 $(\mathrm{S} 21$ ) 在所有采样点中检出浓度最高 $(92.1 \mathrm{ng} / \mathrm{L}), \mathrm{PFOA}$ 浓度达到 $69.3 \mathrm{ng} / \mathrm{L}$, 单体贡献率为 $75.2 \%$. 泰东河是姜堰区最主要的主干河道, 东接溱湖湿地, 西连新通扬运河, 沿岸 居民聚集, 支流汇人口众多, 是该流域农业面源径流和居民生活污水的主要受纳水体, 这可能是引起 PFCs 浓度偏高的主要原因. 溱湖湿地公园平均浓度为 $43.3 \mathrm{ng} / \mathrm{L}$, 显著低于居民聚集区, 略高于其他几个研究区 域. 溱湖湿地公园与泰东河水体连通,在水体交换过程中泰东河水体中的 PFCs 进人到湿地公园, 可能是该 区域 PFCs 相对偏高的原因. 其他研究区域(包括城市区、工业区、农业区、水产养殖区等)浓度相近, PFCs 平 均浓度在 30 40 ng/L 之间, 其中 PFCs 浓度最高的为工业区 (39.8 ng/L), 污染浓度最低的是水产养殖区 (30.4 ng/L). PFNA、PFOS 和 PFDA 3 种单体的最高浓度均出现在居民聚集区或居民/农业混合区. 分析 7 个研究区域中 4 种 PFCs 贡献率, 发现姜堰区所有采样点中 4 种 PFCs 贡献占比具有较高的相关性 (0.999> $\left.R^{2}>0.942, P<0.01\right)$, 这说明姜堰区河湖水系中 PFCs 的污染来源具有相似性. 根据比值法对 PFCs 来源定性 分析 ${ }^{[28]}$, 在整个采样区域 PFOS/PFOA 值均小于 1 , 这表明该区域降雨输人是最主要的污染途径, $42 \%$ 的点 位 PFOA/PFNA 值在 7 15 之间, 36\% 的点位 PFOA/PFNA 值大于 15 , 这表明研究区域 PFCs 污染受工业排 放和前驱体降解的双重作用, 在水产养殖区、溱湖湿地、城市区 PFCs 的来源由前驱体发生降解主导, 其他区 域主要受工业点源排放的影响. 农村或乡镇居民聚集区相对于城市居民聚集区来说, PFCs 污染更为严重, 主要由于农村或乡镇区域的污水收集、处理能力偏弱, 小型工厂零星分布. 另外, 农村或乡镇居民聚集区水 系中 PFOA/PFNA 均值为 7.6, 表明该区点源排放是 PFCs 污染的主导因素, 而湿地公园 PFCs 污染应考量汇 人河流的水质情况.

溱湖湿地是我国著名的国家级生态湿地公园,4 种 PFCs 检出率接近 100\%, 检出总浓度在 $29.4 \sim 50.6 \mathrm{ng} / \mathrm{L}$ 之间, 其中 PFOA 和 PFOS 是最主要的污染物, 浓度范围分别为 $25.0 \sim 38.8 \mathrm{ng} / \mathrm{L}$ (平均浓度 $29.8 \mathrm{ng} / \mathrm{L}$ ) 和 $3.5 \sim$ $9.6 \mathrm{ng} / \mathrm{L}$ (平均浓度 $6.3 \mathrm{ng} / \mathrm{L}$ ). 以 PFOA 和 PFOS 为目标, 表 5 总结了溱湖湿地公园与其他淡水湖泊中两种污 染物的污染水平. 从表 5 可以看出, PFOA 在溱湖湿地中的污染水平显著低于白洋淀 (2010)、武汉的东湖 (2009) 和汤逊湖 (2011), 与巢湖(2015)、太湖流域(2009) 污染水平相当, 显著高于千岛湖 (2017)、深圳水库 (2012)、官厅水库(2008)、鄱阳湖(2011)、意大利 Maggiore Lake(2006) 等水域. PFOS 污染水平显著低于白洋淀 (2010)、武汉东湖(2009) 和汤逊湖 (2011)、太湖(2009)、东莞同沙水库(2017) 和韩国 Shihwa Lake(2004), 与巢 湖(2015)、意大利 Maggiore Lake(2006) 和美国的 Great Lake(2019)污染水平相当, 显著高于鄱阳湖 (2011)、官 厅水库 (2008) 等水域. 总体上溱湖湿地公园中 PFOA 和 PFOS 的污染程度处于中等偏下水平.

表 5 溱湖湿地公园与全球部分地区淡水湖泊 PFOA 和 PFOS 浓度比较*

Tab.5 Comparison of the concentration of PFOA and PFOS between Qinhu Wetland Park and freshwater lakes around the world

\begin{tabular}{|c|c|c|c|c|}
\hline 采样水体 & $\mathrm{PFOA} /(\mathrm{ng} / \mathrm{L})$ & $\mathrm{PFOS} /(\mathrm{ng} / \mathrm{L})$ & 采样时间 & 参考文献 \\
\hline 溱湖 & $25.0 \sim 38.8(29.8)$ & $3.5 \sim 9.6(6.3)$ & 2018 年 & 本研究 \\
\hline 千岛湖 & $0.52 \sim 3.61(1.47)$ & ND & 2017 年 & {$[27]$} \\
\hline 太湖 & $10.6 \sim 36.7(21.7)$ & $3.6 \sim 394(26.5)$ & 2009 年 & {$[29]$} \\
\hline 官厅水库 & $0.55 \sim 2.3$ & $\mathrm{ND} \sim 0.52$ & 2008 年 & {$[30]$} \\
\hline 鄱阳湖 & $0.30 \sim 1.89(1.10)$ & $\mathrm{ND} \sim 0.71(0.35)$ & 2011 年 & {$[31]$} \\
\hline 白洋淀 & $13.6 \sim 441(147)$ & $0.58 \sim 51.2(15.2)$ & 2010 年 & {$[32]$} \\
\hline 武汉东湖 & $15.8 \sim 158(55.0)$ & $9.06 \sim 132(60.4)$ & 2009 年 & {$[33]$} \\
\hline 武汉汤逊湖 & $70.5 \sim 1390(372)$ & $73.4 \sim 1650(357)$ & 2011 年 & {$[34]$} \\
\hline 深圳水库群 & $\mathrm{ND} \sim 7.2(2.4)$ & $\mathrm{ND} \sim 6.3(1.4)$ & 2012 年 & {$[35]$} \\
\hline 东莞同沙水库 & $2.15 \sim 26.70(12.90)$ & $\mathrm{ND} \sim 44.85(14.63)$ & 2017 年 & {$[36]$} \\
\hline 巢湖 & $17.1 \sim 33.3(21.6)$ & $2.37 \sim 6.81(4.29)$ & 2015 年 & {$[37]$} \\
\hline Shihwa Lake(韩国) & $0.9 \sim 62$ & $2.24 \sim 651(17.4)$ & 2004 年 & {$[38]$} \\
\hline Maggiore Lake(意大利) & $1.8 \sim 2.9(2.4)$ & $7.2 \sim 8.6(7.8)$ & 2006 年 & {$[39]$} \\
\hline Great Lake(美国) & $<21$ & $<5.8$ & 2019 年 & {$[40]$} \\
\hline
\end{tabular}

* 括号内为污染物均值, ND 表示没有检出. 


\subsection{PFCs 在胶体相中的分布特征}

胶体广泛存在于地表水环境中,是多种新型有机污染物的重要“汇” “41] 能改变污染物在水环境中的迁 移、转化、生物累积等多种环境行为, 并对水生生物的生长产生重要影响 ${ }^{[42-43]} .4$ 种 PFCs 在胶体介质中的赋 存状况见图 3 和表 4. 在胶体相中,4 种 PFCs 中均被检出, 检出率为 78.8\% 100\%, 其中 PFOA 和 PFOS 检出 率 100\%, PFNA 次之 (97\%). 胶体相中 PFCs 的总浓度范围为 5.4 49.9 ng/L. 与 PFCs 在传统溶解相中的单 体分布相似, PFOA 仍然是最主要的污染物, 浓度范围为 $2.4 \sim 28.0 \mathrm{ng} / \mathrm{L}$, 其次是 PFOS $(0.2 \sim 10.2 \mathrm{ng} / \mathrm{L})$ 、 PFNA $(0 \sim 20.1 \mathrm{ng} / \mathrm{L}) 、 \mathrm{PFDA}(0 \sim 8.9 \mathrm{ng} / \mathrm{L})$, 平均浓度分别为 $11.7 、 3.5 、 2.2$ 和 $1.0 \mathrm{ng} / \mathrm{L}$. 就单体组成而言, 在 胶体相中, PFOA 的平均占比为 $66.6 \%$, 其次是 $\operatorname{PFOS}(19.6 \%) 、 \operatorname{PFNA}(9.9 \%)$ 和 PFDA $(4.0 \%)$. 在太湖流域常 熟地区平原河网中, PFOA、PFOS、PFNA 和 PFDA 的平均浓度分别达到 $113.07 、 47.41 、 15.72$ 和 $40.25 \mathrm{ng} / \mathrm{L}$, 高 于姜堰区 PFCs 在胶体中的赋存水平,但成分组成占比与本文相似 ${ }^{[44]}$.

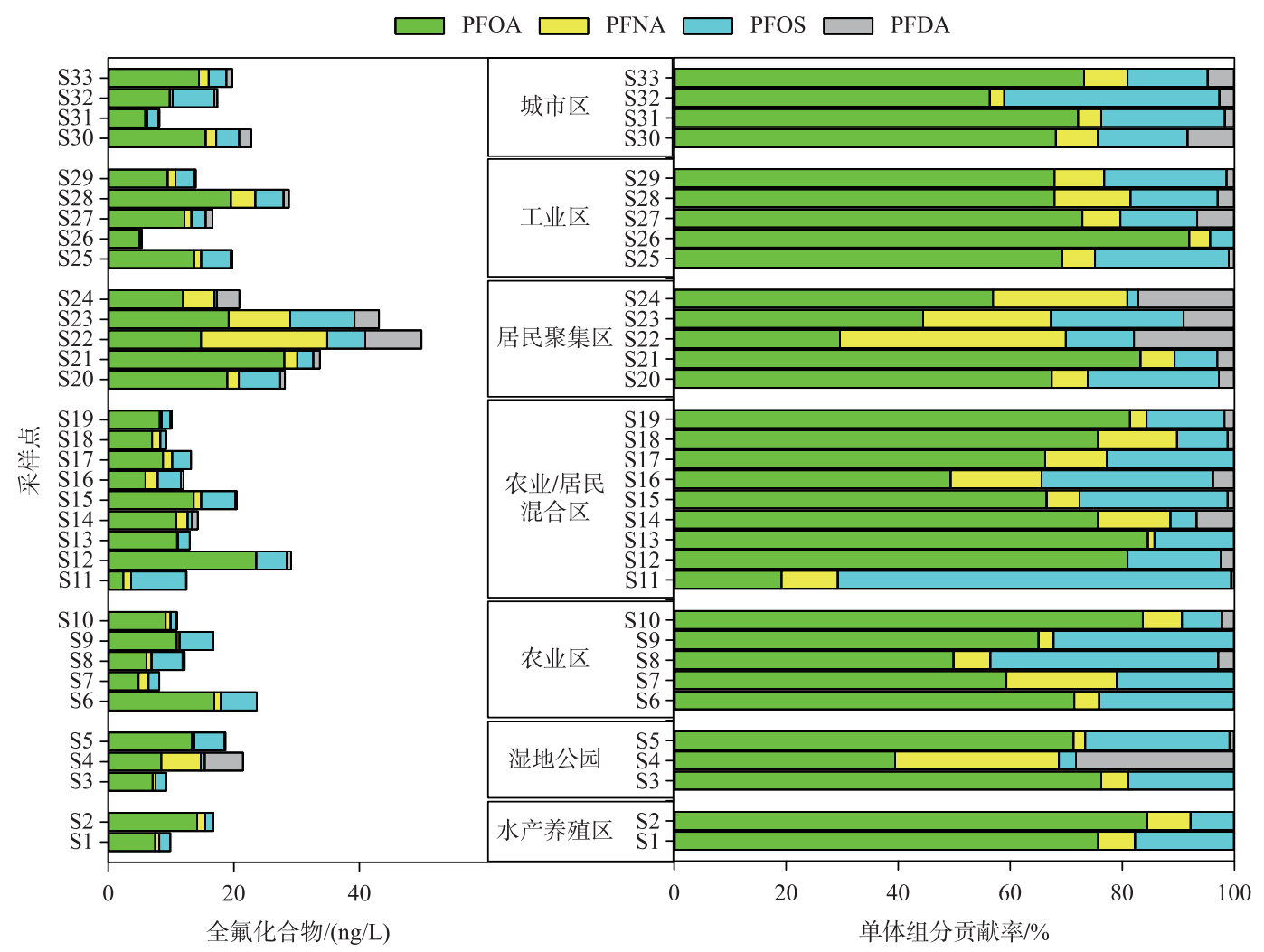

图 3 姜堰区 33 个点位胶体相的 PFCs 浓度与组成

Fig.3 Concentrations and composition of 33 PFCs in colloidal phase of Jiangyan District

从空间分布来看, 居民聚集区地表水体中 PFCs 的赋存水平最高, 总浓度达到 $35.1 \mathrm{ng} / \mathrm{L}$, 其他区域水体 中 PFCs 总浓度相差不大, 浓度范围为 $13.3 \sim 17.0 \mathrm{ng} / \mathrm{L}$, 最高浓度依然出现在姜堰区的主干河流泰东河 (S22), 位于传统溶解相 PFCs 污染水平最高点 (S21) 的上游, 毗邻溱湖湿地公园. 与溶解相相比, 该点位 (S22) 胶体相中的主要 PFCs 组分是 PFNA 和 PFOA, 浓度分别达到 20.1 和 $14.8 \mathrm{ng} / \mathrm{L}$, 单体贡献率分别为 $40.3 \%$ 和 $29.6 \%$, 其次为 PFDA (17.9\%) 和 PFOS (12.2\%), 说明胶体中污染物的组成与溶解相略有区别, 这可 能与污染物物化性质等相关. 从 7 个区域河湖水系中 PFCs 的组成来看, 4 种 PFCs 的贡献占比具有相似性, 除了居民聚集区和城市区中 PFCs 组分占比相关性稍低外 $\left(R^{2}<0.88\right)$, 其他区域组分贡献相关性均大于 0.9 , 这说明在研究区域胶体相中 4 种 PFCs 与传统溶解相具有相似的赋存特性, 而 PFCs 单体贡献率在城市和农 村居民聚集区水系胶体中相关性偏弱的影响机制有待进一步研究. 


\subsection{PFCs 在水体中的胶体吸附特征}

为了评估胶体对 PFCs 的潜在影响, 图 4 显示了胶体在传统溶解相中对 PFCs 的吸附潜能. 传统溶解相 中, 胶体对 4 种 PFCs 的平均吸附贡献率在 42.6\% 66.1\% 之间 (图 4A), 其中胶体对 PFDA 的吸附潜能最大, 吸附贡献率在 35\% 89\% 之间, 平均值为 $66.1 \%$, 其次是 PFNA (均值 $53.5 \%$ )、 PFOA ( 47.0\%) 和 PFOS $(42.6 \%)$. 从空间分布来看, 居民聚集区和水产养殖区水体中胶体对 PFCs 的吸附贡献率最大, 分别达到 $57.4 \%$ 和 56.5\% , 其次是城市区 ( 52.8\%)、工业区 (52.3\%)、农业/居民混合区 (50.3\%)、农业区 (49.5\%) 和湿 地区 $(47.9 \%)$, 这说明农村居民聚集区和水产养殖区水体中胶体对 PFCs 具有更高的吸附潜能, 这可能与该 区域的污废水直排和胶体性能有关. 研究发现污水和养殖废水能够改变水体中胶体的粒径分配, 进而影响 胶体对污染物的吸附潜能 ${ }^{[24]}$. 在太湖流域常熟地区河网系统中, 发现胶体对 PFCs 的吸附贡献率在 $45.46 \%$ $62.59 \%$ 之间 ${ }^{[44]}$, 胶体有机碳含量、颗粒粒径、电荷条件等因素是影响 PFCs 在水一胶体两相分配的主要原因, 而与胶体浓度无明显相关. 与悬浮颗粒物相比, 胶体对 PFCs 具有更高的吸附潜能 ${ }^{[4]]}$, 其吸附潜能比悬浮颗 粒物高 $1 \sim 2$ 个数量级 ${ }^{[45]}$. 相似的结果在其他新型污染物中也被发现, 传统溶解相中 $4 \% \sim 45 \%$ 的药物活性化 合物和 $9.5 \% \sim 50.4 \%$ 的双酚类化合物被胶体介质吸附, 胶体吸附潜能与物质的疏水特性和胶体有机质含量 显著相关 ${ }^{[45-46]}$. 胶体不仅具有较小的粒径、较大的比表面积, 其较高的溶解性有机碳 (DOC) 含量, 更有利于 胶体对有机污染物的吸附作用 ${ }^{[47]}$. 此外, 胶体颗粒还存在 $\mathrm{Ca} 、 \mathrm{Mg} 、 \mathrm{Na}$ 和 $\mathrm{K}$ 等多种元素, 可以通过静电作用和 阳离子交换作用与 PFCs 等有机化合物稳定结合 ${ }^{[28,47]}$. 本研究中胶体对 PFCs 吸附含量占传统溶解相 PFCs 总含量的 $50 \%$ 左右, 胶体对 4 种 PFCs 的吸附贡献率与其正辛醇一水分配系数 ( $\left.\lg K_{\text {ow }}\right)$ 呈现显著的正相关 (图 4B). 这些研究结果表明在传统溶解相中胶体对多种有机污染物具有极强的吸附作用,化合物的疏水性 能是影响水体中 PFCs 赋存和分配的主要因素之一. 胶体粒径较小、结合位点多,极易与有机污染物结合被 水生生物摄取, 且胶体具有较高的生物利用度, 能够改变有机污染物的生物有效性 ${ }^{[48-49]}$, 但胶体及其结合物 生物效应改变的原因尚不明确, 胶体对污染物的吸附机制研究仍需进一步加强.
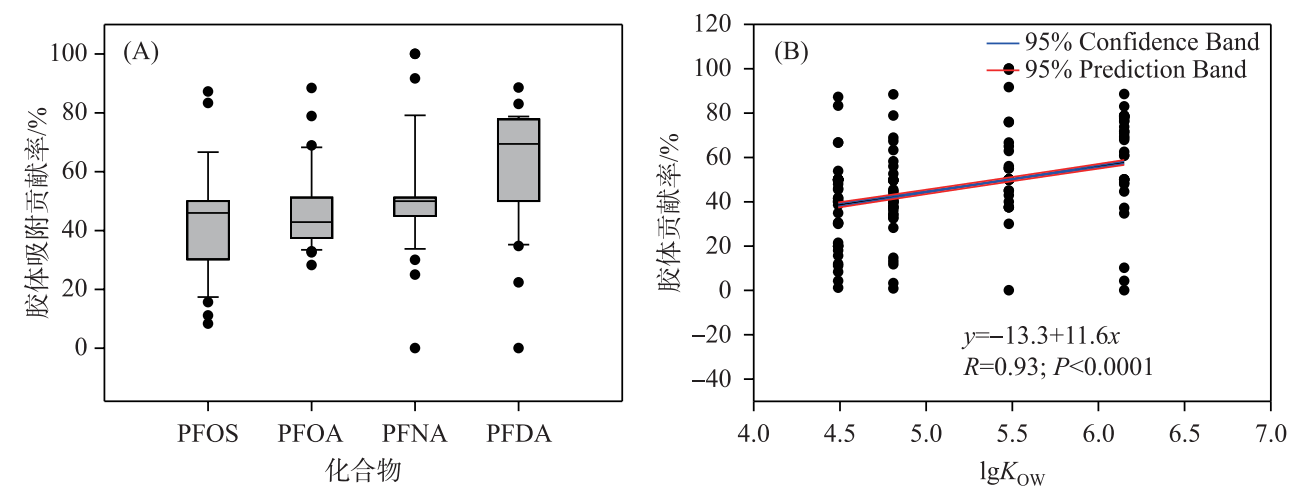

图 4 地表水传统溶解相中胶体对 PFCs 吸附贡献率 $(\mathrm{A})$ 及其与 $\lg K_{\text {ow }}$ 的关系 (B)

Fig.4 Contribution rates of colloids to the PFCs (A) and the relationships between $\lg K_{\mathrm{OW}}$ and contribution rates (B) in traditionally dissolved phase of surface water

\subsection{PFCs 的生态风险评价}

PFCs 对生物具有毒性作用, 长期暴露能够影响幼鱼的性腺发育, 引起雌雄数量失调, 对胚胎生长、生殖 和后代发育产生不利影响, 甚至个体死亡 ${ }^{[50-52]}$. 根据国内外生态风险评价进展, 本研究主要基于熵值法和水 质量基准进行量化表征. 根据本研究区域中 4 种 PFCs 在传统相的检出浓度 $(<70 \mathrm{ng} / \mathrm{L})$ 和评价因子法得到 的 PNEC (3640 ng/L $<P N E C)$ 计算, 发现 PFCs 对藻类、溞类和鱼类的风险水平均低于 0.01 , 这表明该区域内 检出的 PFCs 未对水生生物产生生态风险. 根据物种敏感度分布方法得到的 PNEC $(0.61 \mu \mathrm{g} / \mathrm{L})$ 进行计 算 ${ }^{[53]}$, 发现 PFOS 的生态风险值 $\left(R Q_{\mathrm{PFOS}}\right.$ ) 在 $0.001 \sim 0.034$ 之间, $58 \%$ 的点位大于 0.01 , 处于低风险状态 (图 $5)$. 评估结果表明检出的 PFCs 对于水环境中 $95 \%$ 的生物物种不存在生态风险, 只有少部分生物物种处于低 
风险状态. 物种敏感度分布方法使用的毒理数据主要来源于急性和慢性生态毒性试验的生长率、死亡率等 测试终点, 忽略了 PFCs 长期暴露对生态系统丰度、生产量等方面的影响. 熵值法计算过程中毒理数据单一, 未考虑物种之间的相互关系及污染物的生物富集和放大效应, 但 PFCs 难降解, 持久性强, 且具有较高的生 物富集性, 容易在生物体和食物网中累积放大. 由于胶体对 PFCs 生物有效性的影响机制和贡献量尚不清 楚, 生态风险评价过程未考虑胶体存在对 PFCs 毒理效应的影响. 因此, 在有机污染物的生态风险评估过程 中, 综合考虑胶体存在对有机污染物毒理效应的影响权重和评价方法有效性, 对准确评估水环境中 PFCs 等 有机污染物的生态风险具有重要意义.

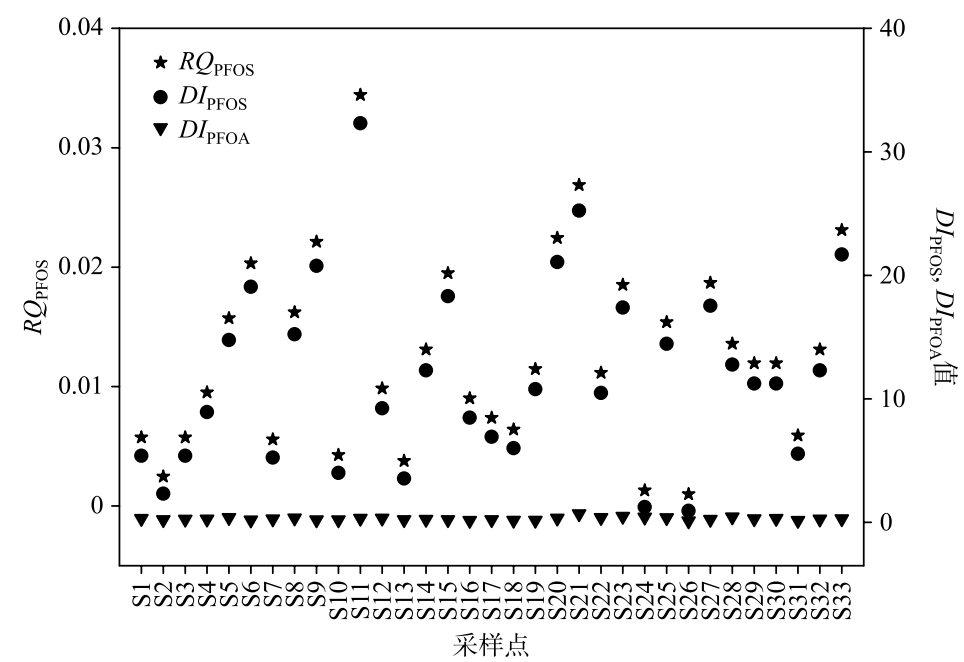

图 5 研究区域传统溶解相中 PFOS 的生态风险值 $\left(R Q_{\mathrm{PFOS}}\right)$ 和 PFOA、PFOS 环境检出浓度与环境质量标准比值 $\left(D I_{\mathrm{PFOA}}\right.$ 和 $\left.D I_{\mathrm{PFOS}}\right)$

Fig.5 $R Q_{\mathrm{PFOS}}, D I_{\mathrm{PFOA}}$ and $D I_{\mathrm{PFOS}}$ of PFOA/PFOS in traditionally dissolved phase of surface water from study area

根据欧盟委员会设定的 PFOS 在淡水环境中的年均环境质量标准 $\mathrm{AA}-\mathrm{EQS}_{\mathrm{fw}}(0.65 \mathrm{ng} / \mathrm{L})$, 所有采样点中

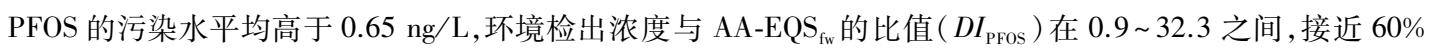
的点位高出此标准 10 倍以上, 最高达到 32 倍左右, 但仍远低于最大允许浓度 $36 \mu \mathrm{g} / \mathrm{L}$, 这表明姜堰区水环 境中 PFOS 对水生生物产生急性风险的概率较低, 但长期慢性风险不容忽视. 这与我国天津滨海新区湿地公 园 PFOS 生态风险水平相似, 湿地公园中 PFOS 的浓度大于 $0.65 \mathrm{ng} / \mathrm{L}$, 低于最大允许浓度 $36 \mu \mathrm{g} / \mathrm{L}^{[22,54]}$. 根 据 Valsecchi 等 ${ }^{[55]}$ 计算的年均环境质量标准 $\mathrm{AA}-\mathrm{EQS}_{\mathrm{fw}}(0.1 \mu \mathrm{g} / \mathrm{L})$, PFOA 在所有采样点中的污染水平均低于 $0.1 \mu \mathrm{g} / \mathrm{L}$, 环境检出浓度与 $\mathrm{AA}^{-E_{\mathrm{S}}} \mathrm{f}_{\mathrm{fw}}$ 的比值 $\left(D I_{\mathrm{PFOA}}\right)$ 在 $0.12 \sim 0.69$ 之间, 在姜堰区主要纳污河流泰东河流域 PFOA 污染水平接近年均环境质量标准值, 存在一定的慢性生态风险, 但均低于 $2220 \mu \mathrm{g} / \mathrm{L}$ 的最大可接受浓 度和 EPA 规定的饮用水中的限值 $(0.4 \mu \mathrm{g} / \mathrm{L})$. 利用 Valsecchi 等提出的海水水生生物的浓度限值 ${ }^{[55]}$, 王世亮 等对山东省 20 个滨海旅游度假区水体中 PFOA 和 PFOS 污染水平进行风险评估, 发现该区域水体中 PFOA 和 PFOS 污染水平均高于欧盟规定的保护海洋生物的浓度限值, PFOS 和 PFOA 造成的生态毒理风险不容忽 视, 建议加强立法与污染管控 ${ }^{[56]}$.

\section{3 结论}

在传统溶解相和胶体相中, PFOA、PFNA、PFOS 和 PFDA 均被检出, 检出率在 $78.8 \% \sim 100 \%$ 之间, 总浓度 范围分别为 12.5 92.1 和 5.4 49.9 ng/L. PFOA 是溶解相和胶体相最主要的 PFCs, 贡献率在 $65 \%$ 以上. 从空 间分布来看, 居民聚集区水域中 PFCs 污染水平最高, 其次是溱湖湿地公园、工业区、农业区及城市区, 水产 养殖区污染水平最低. 在传统溶解相中, 胶体对 PFCs 的平均吸附贡献率在 $42.6 \% \sim 66.1 \%$ 之间, 农村居民聚 
集区和水产养殖区水体中胶体呈现出较高的吸附潜能. 胶体对 PFCs 的吸附贡献率与其疏水特性呈显著正 相关, 传统溶解相中平均 $66.1 \%$ 的 PFDA 吸附在胶体介质中. 从风险评价结果可知, PFOS 对大部分水域中的 水生生物产生低风险毒理影响. 此外, PFOS 的污染水平均高于欧盟委员会设定的 PFOS 在淡水环境中的年 均环境质量标准 $(0.65 \mathrm{ng} / \mathrm{L})$, 且 PFCs 具有累积特性和长距离传输能力, 其长期的累积效应依然不容忽视.

\section{4 参考文献}

[ 1 ] Zhang M, Lou QT, Shao QW et al. Research progress of perfluorinated compounds pollution status and risk assessment. Asian Journal of Ecotoxicology, 2019, 14(3) : 30-53. DOI: 10.7524/AJE.1673-5897.20180821001. [张美, 楼巧婷, 邵 倩文等. 全氟化合物污染现状及风险评估的研究进展. 生态毒理学报, 2019，14(3) : 30-53.]

[2] Elmonznino JA. Occurrence, fate and distribution behaviors of organic contaminants, perfluorinated alkyl acids and phthalic acid esters, in wastewater effluent and the Housatonic River Estuary. Storrs, CT: University of Connecticut, 2016.

[ 3 ] Clara M, Gans O, Weiss S et al. Perfluorinated alkylated substances in the aquatic environment: An Austrian case study. Water Research, 2009, 43(18) : 4760-4768. DOI: 10.1016/j.watres.2009.08.004.

[ 4 ] Yang YL, Lu GH, Yang WX et al. Levels and distribution of perfluorinated compounds in water and biological samples from the Shenyang area, China. Acta Scientiae Circumstantiae, 2010, 30 (10): 2097-2107. DOI: 10.13671/j. hjkxxb. 2010.10.022. [杨永亮, 路国慧, 杨伟贤等. 沈阳地区水环境和生物样品中全氟化合物的污染分布特征. 环境科学 学报, 2010, 30(10): 2097-2107.]

[ 5 ] Yamashita N, Taniyasu S, Petrick G et al. Perfluorinated acids as novel chemical tracers of global circulation of ocean waters. Chemosphere, 2008, 70(7) : 1247-1255. DOI: 10.1016/j.chemosphere.2007.07.079.

[ 6 ] Cao YX, Cao XZ, Wang H et al. Assessment on the distribution and partitioning of perfluorinated compounds in the water and sediment of Nansi Lake, China. Environmental Monitoring and Assessment, 2015, 187 (10) : 1-9. DOI: 10.1007/ s10661-015-4831-9.

[ 7 ] Liu BL, Zhang H, Yu Y et al. Perfluorinated compounds (PFCs) in soil of the Pearl River Delta, China: Spatial distribution, sources, and ecological risk assessment. Archives of Environmental Contamination and Toxicology, 2020, 78(2): 182-189. DOI: 10.1007/s00244-019-00674-1.

[ 8 ] Senthilkumar K, Ohi E, Sajwan K et al. Perfluorinated compounds in river water, river sediment, market fish, and wildlife samples from Japan. Bulletin of Environmental Contamination and Toxicology, 2007, 79(4) : 427-431. DOI: 10. 1007/s00128-007-9243-2.

[ 9 ] Zhang T, Wu Q, Sun HW et al. Perfluorinated compounds in whole blood samples from infants, children, and adults in China. Environmental Science \& Technology, 2010, 44(11): 4341-4347. DOI: 10.1021/es1002132.

[10] Wang TY, Wang P, Meng J et al. A review of sources, multimedia distribution and health risks of perfluoroalkyl acids (PFAAs) in China. Chemosphere, 2015, 129: 87-99. DOI: 10.1016/j.chemosphere.2014.09.021.

[11] Johansson N, Fredriksson A, Eriksson P. Neonatal exposure to perfluorooctane sulfonate (PFOS) and perfluorooctanoic acid (PFOA) causes neurobehavioural defects in adult mice. Neuro Toxicology, 2008, 29(1) : 160-169. DOI: 10.1016/j. neuro.2007.10.008.

[12] Fang XM, Feng YX, Shi ZM et al. Alterations of cytokines and MAPK signaling pathways are related to the immunotoxic effect of perfluorononanoic acid. Toxicological Sciences, 2009, 108(2) : 367-376. DOI: 10.1093/toxsci/kfp019.

[13] Chen CL, Lu YL, Zhang X et al. A review of spatial and temporal assessment of PFOS and PFOA contamination in China. Chemistry and Ecology, 2009, 25(3) : 163-177. DOI: 10.1080/02757540902918321.

[14] Du GY, Jiang XP, Zhuo L et al. Distribution characteristics and risk assessment of perfluorinated compounds in surface water from Chongqing section of the Yangtze River. Ecology and Environmental Sciences, 2019, 28(11) : 2266-2272. [杜 国勇, 蒋小萍, 卓丽等. 长江流域重庆段水体中全氟化合物的污染特征及风险评价. 生态环境学报, 2019, 28 (11) : 2266-2272.]

[15] Li J, Gao Y, Wang ZF et al. Risk assessment of perfluoroalkyl compounds ( PFCs) in water and sediment samples of Hanjiang river. Acta Scientiarum Naturalium Universitatis Pekinensis, 2017, 53(5) : 913-920. DOI: 10.13209/j.0479-8023. 2017.096. [李杰, 高月, 王之芬等. 汉江水体和沉积物中全氟化合物的风险评估. 北京大学学报: 自然科学版, 2017, 53 (5) : 913-920. ] 
[16] Li Z. Distribution and risk assessment of perfluoroalkyl substances in lakes from the middle reach of Yangtze River, China [Dissertation]. Beijing: University of Chinese Academy of Sciences, 2019. [李珍. 长江中游地区湖泊全氟化合物的污 染特征及生态风险评估 [学位论文]. 北京: 中国科学院大学, 2019.]

[17] Sun ZY, Zhang CJ, Yan H et al. Spatiotemporal distribution and potential sources of perfluoroalkyl acids in Huangpu River, Shanghai, China. Chemosphere, 2017, 174: 127-135. DOI: 10.1016/j.chemosphere.2017.01.122.

[18] Zhang DW, Wang DG, Zhang L et al. A study on the perfluorinated compounds pollution in Meiliang Bay, Taihu Lake. Acta Scientiae Circumstantiae, 2012, 32(12) : 2978-2985. DOI: 10.13671/j.hjkxxb.2012.12.016. [张大文, 王冬根, 张莉等. 太湖梅梁湾全氟化合物污染现状研究. 环境科学学报, 2012, 32(12): 2978-2985.]

[19] Liu BL, Zhang H, Xie LW et al. Spatial distribution and partition of perfluoroalkyl acids (PFAAs) in rivers of the Pearl River Delta, Southern China. Science of the Total Environment, 2015, 524/525: 1-7. DOI: 10.1016/j. scitotenv.2015. 04.004.

[20] Shi R, Mao RY, Zhang M et al. Distribution, sources, and ecological risks of polyfluoroalkyl substances in the surface water of the wuliangsuhai watershed. Environmental Science, 2021, 42(2) : 663-672. DOI: 10.13227/j.hjkx. 202005216. [史锐, 毛若愚, 张梦等. 乌梁素海流域地表水中全氟化合物分布、来源及其生态风险. 环境科学, 2021, 42(2): 663-672.]

[21] Lee YM, Lee JY, Kim MK et al. Concentration and distribution of per- and polyfluoroalkyl substances (PFAS) in the Asan Lake area of South Korea. Journal of Hazardous Materials, 2020, 381: 120909. DOI: 10. 1016/j. jhazmat. 2019.120909.

[22] Navarro I, de la Torre A, Sanz P et al. Perfluoroalkyl acids (PFAAs): Distribution, trends and aquatic ecological risk assessment in surface water from Tagus River basin (Spain). Environmental Pollution, 2020, 256: 113511. DOI: 10.1016/ j.envpol.2019.113511.

[23] Li YN, Xu HC, Jiang HL. Molecular weight distribution, fluorescence characteristics of dissolved organic matter and their effect on the distribution of heavy metals of Lake Poyang. J Lake Sci, 2020, 32(4) : 1029-1040. DOI: 10.18307/2020. 0412. [李雅妮, 徐华成, 江和龙. 鄱阳湖水体溶解有机质分子量分布、苂光特征及对重金属分布的影响. 湖泊科 学, 2020, 32(4): 1029-1040.

[24] Yan CX. Effect of aquatic colloids on thebehavior of selected emerging organic contaminants in the Yangtze Estuary[Dissertation]. Shanghai : East China Normal University, 2014. [晏彩霞. 长江口滨岸水中胶体对新兴有机污染物环境行为 的影响研究 [学位论文]. 上海: 华东师范大学, 2014.]

[25] Durjava MK, Kolar B, Peijnenburg W. Case studies on the development and application of in-silico techniques for environmental hazard and risk assessment. National Institute of Public Health and the Environment (RIVM), Laboratory for Ecological Risk Assessment, 2012.

[26] Han TZ. Distribution and potential sources of perfluoroalkyl substances (PFASs) in Jiaozhou bay[ Dissertation]. Qingdao: First Institute of Cceanography, SOA, 2018. [韩同竹. 胶州湾海水和生物体中全氟化合物的分布特征及来源解析 [学位论文]. 青岛: 国家海洋局第一海洋研究所, 2018.]

[27] Zhang M, Tang FL, Cheng XL et al. Occurrence and distribution of perfluorinated compounds in surface water of Lake Qiandao(Xin'anjiang Reservoir). J Lake Sci, 2020, 32(2) : 337-345. DOI: 10.18307/2020.0204. [张明, 唐访良, 程新 良等. 千岛湖 (新安江水库) 表层水中全氟化合物的残留水平及分布特征. 湖泊科学, 2020, 32(2): 337-345.]

[28] Wang TY, Khim JS, Chen CL et al. Perfluorinated compounds in surface waters from Northern China: Comparison to level of industrialization. Environment International, 2012, 42 : 37-46. DOI: 10.1016/j.envint.2011.03.023.

[29] Yang LP, Zhu LY, Liu ZT. Occurrence and partition of perfluorinated compounds in water and sediment from Liao River and Taihu Lake, China. Chemosphere, 2011, 83(6) : 806-814. DOI: 10.1016/j.chemosphere.2011.02.075.

[30] Wang TY, Chen CL, Naile JE et al. Perfluorinated compounds in water, sediment and soil from Guanting reservoir, China. Bulletin of Environmental Contamination and Toxicology, 2011, 87(1) : 74-79. DOI: 10.1007/s00128-011-0307-y.

[31] Zhang DW, Zhang L, Wei YH et al. Investigation of perfluorooctanoic acid(pfoa) and perfluorooctane sulfonate(pfos) pollution in surface water of Poyang lake. Resources and Environment in the Yangtze Basin, 2012, 21(7) : 885-890. [ 张大 文, 张莉, 魏益华等. 鄱阳湖表层水中全氟辛酸和全氟辛烷磺酸污染现状调查. 长江流域资源与环境, 2012,21 (7) : 885-890.]

[32] Zhou Z, Shi YL, Li WH et al. Perfluorinated compounds in surface water and organisms from Baiyangdian lake in North 
China: Source profiles, bioaccumulation and potential risk. Bulletin of Environmental Contamination and Toxicology, 2012, 89(3) : 519-524. DOI: 10.1007/s00128-012-0745-1.

[33] Chen J, Wang LL, Zhu HD et al. Spatial distribution of perfluorooctanoic acids and perfluorinate sulphonates in surface water of east lake. Environmental Science, 2012, 33(8) : 2586-2591. DOI: 10.13227/j.hjkx.2012.08.035. [陈静, 王琳 玲, 朱湖地等. 东湖表层水体中全氟辛酸和全氟辛磺酸空间分布特征. 环境科学, 2012, 33(8): 2586-2591.]

[34] Zhou Z, Liang Y, Shi YL et al. Occurrence and transport of perfluoroalkyl acids (PFAAs), including short-chain PFAAs in Tangxun lake, China. Environmental Science \& Technology, 2013, 47(16) : 9249-9257. DOI: 10.1021/es402120y.

[35] Wang XX, Zhang H, He L et al. Distribution of perfluorinated compounds in surface water of Shenzhen reservoir groups. Environmental Science, 2014, 35(6) : 2085-2090. DOI: 10.13227/j.hjkx.2014.06.007. [王釒塹璇, 张鸿, 何龙等. 深圳 水库群表层水中全氟化合物的分布特征. 环境科学, 2014, 35(6) : 2085-2090.]

[36] Zhang JJ, Xue XY, Huang CS et al. Survey of perfluorooctanoic acid and perfluorooctane sulfonate in surface water from Tongsha Reservoir of Pearl River Delta, South China. Environmental Chemistry, 2017, 36(12) : 2600-2608. [张佳䩀, 薛晓燕, 黄楚珊等. 珠江三角洲同沙水库全氟辛酸和全氟辛烷磺酸污染现状调查. 环境化学, 2017, 36(12): 2600-2608.]

[37] Pan X, Ye J, Zhang H et al. Occurrence, removal and bioaccumulation of perfluoroalkyl substances in lake Chaohu, China. International Journal of Environmental Research and Public Health, 2019, 16(10): E1692. DOI: 10.3390/ijerph16101692.

[38] Rostkowski P, Yamashita N, So IMK et al. Perfluorinated compounds in streams of the Shihwa industrial zone and Lake Shihwa, South Korea. Environmental Toxicology and Chemistry, 2006, 25(9) : 2374-2380. DOI: 10.1897/05-627R.1.

[39] Loos R, Wollgast J, Huber T et al. Polar herbicides, pharmaceutical products, perfluorooctanesulfonate (PFOS), perfluorooctanoate (PFOA), and nonylphenol and its carboxylates and ethoxylates in surface and tap waters around Lake Maggiore in Northern Italy. Analytical and Bioanalytical Chemistry, 2007, 387(4) : 1469-1478. DOI: 10.1007/s00216-0061036-7.

[40] Remucal CK. Spatial and temporal variability of perfluoroalkyl substances in the Laurentian Great Lakes. Environmental Science Processes \& Impacts, 2019, 21(11) : 1816-1834. DOI: 10.1039/c9em00265k.

[41] Yan CX, Nie MH, Yang Y et al. Effect of colloids on the occurrence, distribution and photolysis of emerging organic contaminants in wastewaters. Journal of Hazardous Materials, 2015, 299: 241-248. DOI: 10.1016/j.jhazmat.2015.06.022.

[42] Bai YY, Zheng MF, Zheng AR et al. Effects of nature colloids on the growth of nature bacteria community. Journal of Xiamen University: Natural Science, 2007, 46(4) : 550-554. [白艳艳, 郑敏芳, 郑爱榕等. 天然胶体对天然菌群生长的 影响. 厦门大学学报: 自然科学版, 2007, 46(4) : 550-554.]

[43] Wang F, Zhu G, Xu H et al. The bioeffect of natural colloids on the growth of Microcystis aeruginosa in Lake Taihn, China. China Environmental Science, 2009, 29(5): 538-542. [王芳, 朱广伟, 许海等. 太湖梅梁湾水体中胶体对铜绿微 囊藻生长的促进效应. 中国环境科学, 2009, 29(5) : 538-542.]

[44] Li XQ, Hua ZL. Multiphase distribution and spatial patterns of perfluoroalkyl acids (PFAAs) associated with catchment characteristics in a plain river network. Chemosphere, 2021, 263: 128284. DOI: 10.1016/j.chemosphere.2020.128284.

[45] Duan YP, Meng XZ, Wen ZH et al. Multi-phase partitioning, ecological risk and fate of acidic pharmaceuticals in a wastewater receiving river: The role of colloids. Science of the Total Environment, 2013, 447: 267-273. DOI: 10.1016/j. scitotenv.2013.01.017.

[46] Si W, Cai YF, Liu JC et al. Investigating the role of colloids on the distribution of bisphenol analogues in surface water from an ecological demonstration area, China. Science of the Total Environment, 2019, 673: 699-707. DOI: 10.1016/j. scitotenv.2019.04.142.

[47] Pan B, Qiu MY, Wu M et al. The opposite impacts of Cu and Mg cations on dissolved organic matter-ofloxacin interaction. Environmental Pollution, 2012, 161: 76-82. DOI: 10.1016/j.envpol.2011.09.040.

[48] Sun Y, Liu JC, Lu GH. Influence of aquatic colloids on the bioaccumulation and biological effects of diclofenac in zebrafish (Danio rerio). Ecotoxicology and Environmental Safety, 2020, 195: 110470. DOI: 10. 1016/j. ecoenv. 2020.110470.

[49] Liu WX, He W, Wu JY et al. Effects of fluorescent dissolved organic matters (FDOMs) on perfluoroalkyl acids (PFAAs) in lake and river water. Science of the Total Environment, 2019, 666: 598-607. DOI: 10.1016/j.scitotenv.2019.02.219. 
[50] Olsen GW, Mair DC, Lange CC et al. Per- and polyfluoroalkyl substances (PFAS) in American Red Cross adult blood donors, 2000-2015. Environmental Research, 2017, 157: 87-95. DOI: 10.1016/j.envres.2017.05.013.

[51] Stein CR, McGovern KJ, Pajak AM et al. Perfluoroalkyl and polyfluoroalkyl substances and indicators of immune function in children aged 12-19 y: National Health and Nutrition Examination Survey. Pediatric Research, 2016, 79(2) : 348-357. DOI: $10.1038 /$ pr.2015.213.

[52] Joensen UN, Bossi R, Leffers H et al. Do perfluoroalkyl compounds impair human semen quality? Environmental Health Perspectives, 2009, 117(6) : 923-927. DOI: 10.1289/ehp.0800517.

[53] Qi P, Wang Y, Mu JL et al. Aquatic predicted no-effect-concentration derivation for perfluorooctane sulfonic acid. Environmental Toxicology and Chemistry, 2011, 30(4) : 836-842. DOI: 10.1002/etc.460.

[54] Chen ZA, Ren GB, Ma XD et al. Perfluoroalkyl substances in the Lingang hybrid constructed wetland, Tianjin, China: Occurrence, distribution characteristics, and ecological risks. Environmental Science and Pollution Research, 2020,27 (31) : 38580-38590. DOI: 10.1007/s11356-020-09921-8.

[55] Valsecchi S, Conti D, Crebelli R et al. Deriving environmental quality standards for perfluorooctanoic acid (PFOA) and related short chain perfluorinated alkyl acids. Journal of Hazardous Materials, 2017, 323: 84-98. DOI: 10.1016/j.jhazmat.2016.04.055.

[56] Wang SL, Cao XZ. Contamination characteristics and potential ecological risks of typical perfluoroalkyl substances in the water and sediment of coastal tourism resorts in Shandong Province. Environmental Science, 2020, 41 (12) : 5428-5437. DOI : 10.13227/j.hjkx.202003286. [王世亮, 曹雪稚. 山东省滨海旅游度假区水体环境典型全氟化合物污染特征及 潜在生态风险. 环境科学, 2020, 41(12) : 5428-5437.] 